\title{
Lithiation of silicon via lithium Zintl-defect complexes from first principles
}

\author{
Andrew J. Morris, ${ }^{1, *}$ R. J. Needs, ${ }^{2}$ Elodie Salager, ${ }^{3}$ C. P. Grey, ${ }^{3}$ and Chris J. Pickard ${ }^{1}$ \\ ${ }^{1}$ Department of Physics and Astronomy, University College London, Gower St, London WC1E 6BT, United Kingdom \\ ${ }^{2}$ Theory of Condensed Matter Group, Cavendish Laboratory, University of Cambridge, J. J. Thomson Avenue, \\ Cambridge CB3 OHE, United Kingdom \\ ${ }^{3}$ Department of Chemistry, University of Cambridge, Lensfield Road, Cambridge CB2 1EW, United Kingdom
}

(Received 26 November 2012; revised manuscript received 22 March 2013; published 21 May 2013)

\begin{abstract}
An extensive search for low-energy lithium defects in crystalline silicon using density-functional-theory methods and the ab initio random structure searching (AIRSS) method shows that the four-lithium-atom substitutional point defect is exceptionally stable. This defect consists of four lithium atoms with strong ionic bonds to the four under-coordinated atoms of a silicon vacancy defect, similar to the bonding of metal ions in Zintl phases. This complex is stable over a range of silicon environments, indicating that it may aid amorphization of crystalline silicon and form upon delithiation of the silicon anode of a Li-ion rechargeable battery.
\end{abstract}

DOI: 10.1103/PhysRevB.87.174108

PACS number(s): 61.05.-a, 61.72.J-, 82.47.Aa

Monovacancies in silicon may be retained after growth with concentrations of about $3 \times 10^{16} \mathrm{~cm}^{-3} \cdot{ }^{1}$ Lithium present in the bulk is strongly attracted to vacancies, although there is a substantial energy barrier to forming a lithium substitutional defect in the perfect crystal. ${ }^{1}$ In this article we denote a defect complex by listing its constituents between braces $(\{\ldots\})$. For example, the lithium substitutional defect is denoted by $\{\mathrm{Li}, V\}$ since it comprises a lithium atom within a silicon vacancy. A lithium-vacancy complex was assigned to an electron paramagnetic resonance (EPR) center by Goldstein ${ }^{2}$ and lithium drift rate experiments have also shown that lithium binds to vacancies, preventing further mobility. ${ }^{3}$ Our density-functional-theory (DFT) calculations show that the formation of a Frenkel-type impurity defect $(\{\mathrm{Li}, V, I\})$ from a $T_{d}$ interstitial Li atom requires an energy of $3.73 \mathrm{eV}$, which is in excellent agreement with the value of $3.75 \mathrm{eV}$ calculated by Wan et $a l .{ }^{4}$ Wan et al. suggested that the substitutional lithium defect, $\{\mathrm{Li}, V\}$ will not form due to its large formation energy. However, we calculate its formation energy from bulk silicon and lithium metal to be $3.09 \mathrm{eV}$, and hence $\{\mathrm{Li}, V\}$ is more stable than a separated vacancy and lithium interstitial $(\{V\}$ and $\{\mathrm{Li}\})$. It is likely that silicon vacancies play a significant role in the interaction between lithium and silicon.

Along with the wide range of technological uses of silicon, such as semiconductor devices and photovoltaics, it has recently been proposed as an anode for lithium-ion batteries. Lithium intercalated graphite is the standard lithium-ion battery (LIB) negative electrode material, due to its good rate capability and cyclability, but demand for even higher performance LIBs has motivated the investigation of other materials. Silicon is an attractive alternative since it has 10 times the gravimetric and volumetric capacity of graphite (calculated from the initial mass and volume of silicon) but, unlike graphite, silicon undergoes structural changes on lithiation. ${ }^{5-7}$

In the first stages of lithiation, in which silicon atoms greatly outnumber lithium, it has been proposed that lithiation occurs as interstitial lithium defects form near the silicon surface. ${ }^{8}$ Previous theoretical studies have shown that a single lithium atom in bulk silicon resides at the $T_{d}$ site and that at higher concentrations lithium clusters can promote the breaking of silicon-silicon bonds. ${ }^{4,9,10}$ There are equal numbers of $T_{d}$ sites and silicon atoms in $c$-Si and, since $a-\mathrm{Li}_{y} \mathrm{Si}$ forms at $y \approx 0.3$ in micron-sized silicon clusters (a Li:Si ratio of $\approx 1: 3^{11,12}$ ), the presence of lithium must lead to the breaking of silicon-silicon bonds before all $T_{d}$ sites are filled with lithium. Chan et al. presented a density-functional-theory study of the lithiation of silicon surfaces and $c-\mathrm{Si}$ in which they described the charging or discharging of the half-cell by the addition or subtraction of a succession of lithium impurities. ${ }^{13}$ Their study suggests a lithiation pathway from $c$-Si to $\mathrm{Li}_{15} \mathrm{Si}_{4}$ and correctly predicts enhanced expansion in the (110) direction, as observed by Goldman et al. ${ }^{14}$ Understanding $\mathrm{Li}$ defects in silicon is a vital step towards a full appreciation of the lithiation of a Si electrode in a LIB.

Our previous study showed that interstitial lithium binds to any hydrogen present and breaks silicon-silicon bonds. ${ }^{15}$ The dynamics of lithium in the bulk are beyond the scope of this study, but understanding the underlying stable lithium configurations is crucial to any subsequent dynamical study. In this article we show that the $\{4 \mathrm{Li}, V\}$ complex is the most stable defect when silicon vacancies are present. Below we describe briefly our computational search method, followed by a description of the $\{4 \mathrm{Li}, V\}$ defect and its behavior in silicon with a range of silicon environments and lithium chemical potentials.

$A b$ initio random structure searching (AIRSS) ${ }^{16}$ has been successful in predicting the ground-state structures of point defects (including the interaction of impurities with vacancies) in semiconductors ${ }^{17,18}$ and ceramics. ${ }^{19,20}$ The initial structures for the searches were prepared as follows. A silicon atom and its four nearest neighbors were removed from a periodic supercell of diamond-structure $(F d \overline{3} m)$ silicon containing 32 atomic sites; this has previously been shown to be large enough to accommodate impurities of this size at the searching stage. ${ }^{17}$ Four silicon atoms, along with either one, two, three, four, or five lithium atoms, were then randomly placed within a sphere of radius $4 \AA$ centered on an atomic site. We relaxed approximately 400 different starting structures. Each structure was relaxed, while keeping the supercell fixed, until the DFT forces on the ions were smaller than $0.05 \mathrm{eV} / \AA$. A few 


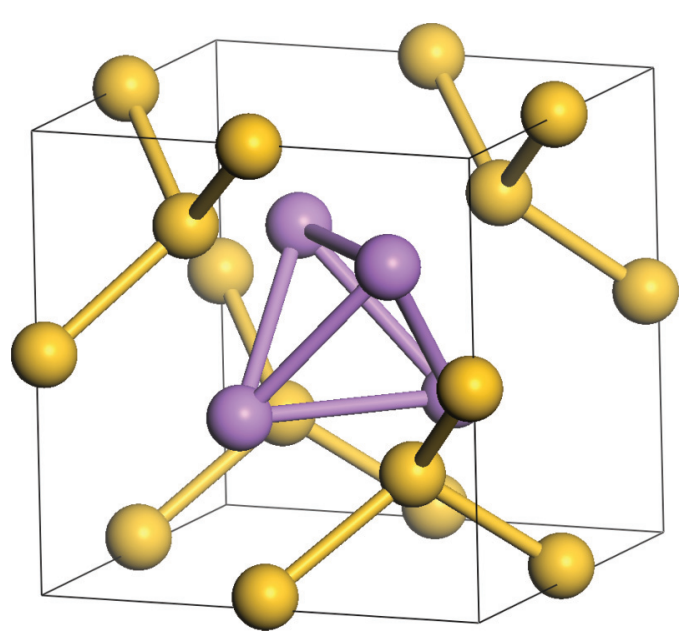

FIG. 1. (Color online) The $\{4 \mathrm{Li}, V\}$ defect of $T_{d}$ symmetry in an eight-atom conventional cell. The lithium atoms form a tetrahedron within the silicon vacancy. Lithium atoms are in pink (dark gray), silicon in yellow (light gray). The bonds between the lithium atoms are to guide the eye.

searches were also carried out adding hydrogen atoms along with lithium over a range of $\mathrm{Li} / \mathrm{H}$ combinations.

The plane wave CASTEP ${ }^{21}$ DFT code and the Perdew-BurkeErnzerhof (PBE) exchange-correlation functional were used with "ultrasoft" pseudopotentials and a basis set containing plane waves with energies of up to $300 \mathrm{eV}$. The Brillouin zone (BZ) was sampled using $2 \times 2 \times 2 k$ points arranged according to the multiple- $k$-point generalization of the Baldereschi scheme. ${ }^{17}$ The candidate structures were then further relaxed to higher accuracy in larger supercells containing 256 atomic sites and a standard $2 \times 2 \times 2$ Monkhorst-Pack (MP) grid corresponding to a BZ sampling grid finer than $2 \pi \times 0.033 \AA^{-1}$.

We found, in order of increasing stability, $\{\mathrm{Li}, V\},\{2 \mathrm{Li}, V\}$, $\{3 \mathrm{Li}, V\}$, and $\{4 \mathrm{Li}, V\}$ complexes. The $\{4 \mathrm{Li}, V\}$ defect with $T_{d}$ symmetry, shown in Fig. 1, was the lowest energy $\mathrm{Li} / \mathrm{Si}$-vacancy complex found. This complex was re-computed allowing spin polarization, but no spin moment was obtained with the PBE density functional. The four lithium atoms are at the vertices of a tetrahedron, with edges of $2.79 \AA$ so that the $\mathrm{Li}$-Li bonds are longer and therefore weaker than those in the lithium dimer $(2.672 \AA)$ but shorter than those in bulk lithium $(2.98 \AA)$. Each lithium atom has three nearest-neighbor (NN) silicon atoms at a distance of $2.51 \AA$ and three second-NNs $2.71 \AA$ away, compared to first and second NNs for the relaxed \{Li\} defect at $2.46 \AA$ and $2.75 \AA$, respectively. ${ }^{9}$ Canham $^{22}$ associated the photoluminescent " $Q$ " line of lithiated silicon with a $\{4 \mathrm{Li}, V\}$ defect, and reported a symmetry lowering to $C_{3 v}$, but we did not find any $\{4 \mathrm{Li}, V\}$ complexes of this symmetry in our AIRSS searches or by breaking the $T_{d}$ symmetry "by hand."

Using the gauge-including projector-augmented wave method (GIPAW) ${ }^{23,24}$ implemented in CASTEP the chemical shift of lithium in the $\{4 \mathrm{Li}, V\}$ complex is $9 \mathrm{ppm}$. This makes the $\{4 \mathrm{Li}, V\}$ complex very difficult to differentiate from the lithium clusters that form in $a-\mathrm{Li}_{y} \mathrm{Si}$, which have broad peaks in the chemical shift range +20 to $-10 \mathrm{ppm} .{ }^{11}$ The isotropic chemical shielding of lithium in $\{4 \mathrm{Li}, V\}$ was obtained in the 32-silicon-atomic-site simulation cell using a BZ sampling finer than $2 \pi \times 0.03 \AA^{-1}$ and a well-converged basis set containing plane waves up to $850 \mathrm{eV}$. The chemical shift was obtained by comparing to the calculated value in crystalline LiF which has a known chemical shift of $\delta=-1 \mathrm{ppm}$ relative to the standard $1 \mathrm{M} \mathrm{LiCl}$ solution (at $0 \mathrm{ppm}$ ). In silicon there is a significant change in lattice constant depending on the exchange-correlation functional chosen. However, using the LDA, GGA, and experimental lattice parameters changed the lithium chemical shift of the $\{4 \mathrm{Li}, V\}$ defect by less than 2 ppm.

The lithium and silicon atoms in the $\{4 \mathrm{Li}, V\}$ complex bond in a similar way to a Zintl phase. Zintl phases consist of an alkali metal or alkaline-earth metal and an element from the $p$-block of the periodic table known as the Zintl ion. The ions are bound electrostatically as the metal ion is completely ionized by the more electronegative Zintl ion. A Zintl ion with atomic number $Z_{\mathrm{zi}}$, which gains $n$ electrons from the metal, requires the donation of $n$ fewer electrons through covalent bonding to satisfy the "octet rule." The Zintl ion behaves isoelectronically with the element of atomic number $Z_{\mathrm{zi}}+n$. For example, the phosphorus $(Z=15)$ atoms in $\mathrm{Li}_{1} \mathrm{P}_{1}$ gain one electron and form long twofold coordinated chains similar to those found in sulfur $(Z=16)$ compounds. ${ }^{25}$

As in the lithium/phosphorus model compounds mentioned above, Mulliken population analysis shows that the lithium atoms in $\{4 \mathrm{Li}, V\}$ have a positive charge, and the valence charge density shows a lone pair of electrons on the four threefold covalently bonded silicon atoms (in this case making the four silicon atoms isoelectronic to phosphorus). The lithium-silicon interaction is not well described as a covalent bond, since each lithium atom has three silicon $\mathrm{NN}$ each with a small $(0.18|e|)$ charge overlap. We believe that the fulfillment of the Zintl rules leads to the stability of the $\{4 \mathrm{Li}, V\}$ defect.

The single silicon vacancy $\{V\}$ has an unoccupied electronic state in the band gap; in contrast $\{4 \mathrm{Li}, V\}$ has a similar-shaped density of electronic states (DOS) but with the gap state filled (see Fig. 2). Projecting the DOS onto local orbitals using OPTADOS reveals that the gap states have a siliconlike character, indicating charge transfer from lithium to silicon. ${ }^{26}$

A selection of charged cells $(2-, 1-, 1+$, and $2+)$ were considered in supercells containing 8, 64, 216, and 512 silicon atomic sites. The atomic positions were optimized after initially breaking the spin symmetry and adding a neutralizing background charge. The charge did not localize around the defect in any of these calculations, indicating that the charged states are unstable, and the neutral defect is therefore the only stable state of $\{4 \mathrm{Li}, V\}$.

We now turn our attention to the energetics of $\{4 \mathrm{Li}, V\}$. The formation energy per atom of the defect is calculated as

$$
E_{f} / A=\left(E-4 \mu_{\mathrm{Li}}-n_{\mathrm{Si}} \mu_{\mathrm{Si}}\right) / 4,
$$

where $E$ is the total energy of the defect cell, $n_{\mathrm{Si}}$ is the number of silicon atoms present in the supercell, and $\mu_{\mathrm{Li}}$ and $\mu_{\mathrm{Si}}$ are the chemical potentials of lithium and silicon, respectively, which are discussed in detail below. When considering the energetics of a defect it is important to compare its stability against physically meaningful chemical potentials. We consider three scenarios: (i) We model silicon prior to lithiation, and therefore both $\mu_{\mathrm{Si}}$ and $\mu_{\mathrm{Li}}$ are derived from their bulk elemental compounds; (ii) on commencement of lithiation the silicon 


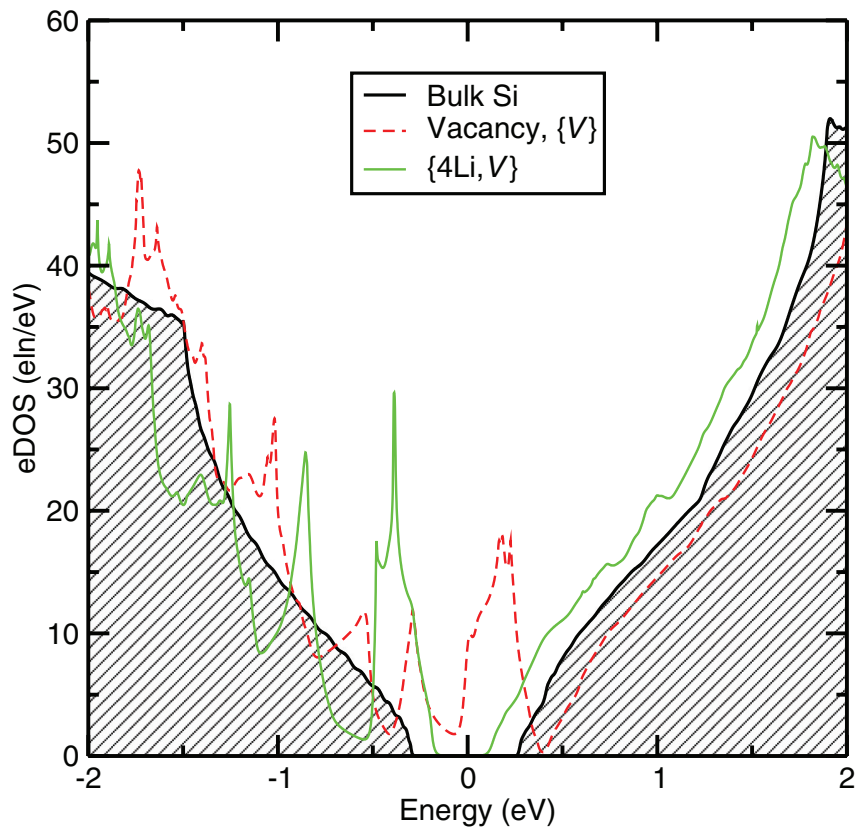

FIG. 2. (Color online) The electronic density of states of the $\{4 \mathrm{Li}, V\}$ defect around the Fermi level (set to $0 \mathrm{eV}$ ) calculated using OPTADOS (green line). The $\{V\}$ defect (red dotted) forms a state in the band gap which is subsequently filled by the electrons donated from the lithium atoms.

contains mobile lithium atoms, therefore $\mu_{\mathrm{Si}}$ is derived from bulk $\mathrm{Si}$ and $\mu_{\mathrm{Li}}$ from the $\{\mathrm{Li}\}$ defect at the $T_{d}$ site; and (iii) is as (i) but, since monovacancies may be found in bulk silicon, $\mu_{\mathrm{Si}}$ is derived from bulk $\mathrm{Si}$ with an isolated vacancy and $\mu_{\mathrm{Li}}$ is taken from Li metal. A $4 \times 4 \times 4 \mathrm{MP}$ grid was used for calculating the defect formation energies in the 256 atom cells, which gives a $k$-point spacing of less than $2 \pi \times 0.016 \AA^{-1}$. The formation energy of the $T_{d}\{4 \mathrm{Li}, V\}$ complex in scenario (i) is $0.02 \mathrm{eV}$ per $\mathrm{Li}$ atom, which is a small positive formation energy, so it seems likely that this complex will form at room temperature. In the type (ii) scenario the formation energy is $-0.30 \mathrm{eV}$ per Li atom, as shown on the left-hand scale of Fig. 3, (the difference in formation energy between the $\{\mathrm{Li}\}$ and $\{4 \mathrm{Li}, V\}$ defects in diamond-structure silicon), implying that it is energetically favorable for mobile lithium atoms present in the bulk to "dig out" a silicon vacancy.

Scenario (iii) requires the calculation of the structure and energetics of the silicon vacancy. When using a simulation cell of 256 sites or more we found that the single silicon vacancy $\{V\}$ lowers its symmetry from $T_{d}$ to $D_{2 d} \cdot{ }^{27-30}$ We calculate the formation energy of $\{V\}$ from bulk silicon to be $3.43 \mathrm{eV}$, which is in excellent agreement with previous calculations. ${ }^{29,30}$ It is energetically favorable for lithium in the form of $\{\mathrm{Li}\}$ and lithium metal to bind strongly to silicon vacancies already present in the crystal. As shown on the right-hand scale of Fig. 3, the $\{4 \mathrm{Li}, V\}$ complex in scenario (iii) has a formation energy of $-0.84 \mathrm{eV}$ per $\mathrm{Li}$ atom, and $-1.15 \mathrm{eV}$ per $\mathrm{Li}$ atom when formed from the $\{V\}$ and $T_{d}$ symmetry interstitial $\{\mathrm{Li}\}$ complex (calculated as the difference between the formation energy of $\{\mathrm{Li}\}$ in bulk silicon and $\{4 \mathrm{Li}, V\}$ in silicon with a $\{V\}$ already present).

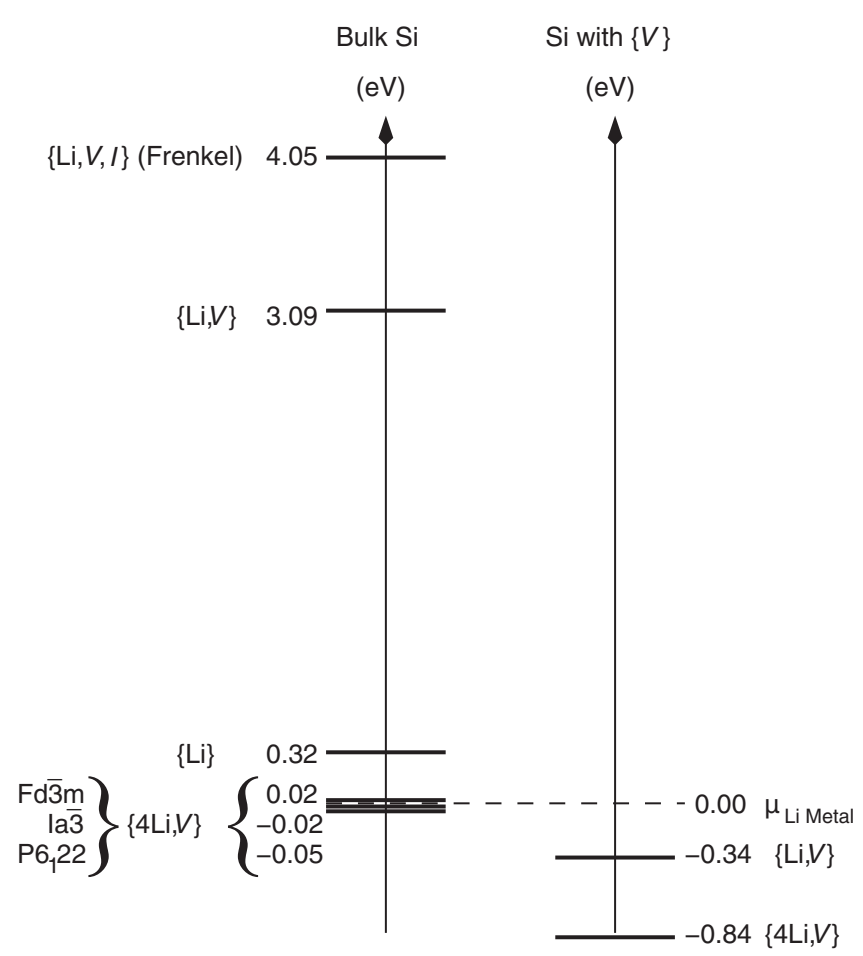

FIG. 3. The chemical potential of lithium in point defects in silicon relative to lithium metal, $\mu_{\mathrm{Li} \text { metal }}$. Any complex lower in energy than $\mu_{\mathrm{Li} \text { metal }}$ is likely to form in silicon from a reservoir of lithium metal and therefore may be found in silicon electrodes on initial lithiation. The left-hand scale shows the chemical potential of lithium defects in bulk silicon; the right-hand scale shows the chemical potential of lithium defects when vacancies are also present in the silicon lattice. The lithium substitutionals $\{\mathrm{Li}, V, I\}$ and $\{\mathrm{Li}, V\}$ are unlikely to form in bulk silicon due to their high energy, but lithium will bind to vacancies present in the bulk forming $\{\mathrm{Li}, V\}$ (see right-hand scale). The most likely complex to form, whether or not vacancies are present, is $\{4 \mathrm{Li}, V\}$. The $\{4 \mathrm{Li}, V\}$ complex is almost degenerate with $\mu_{\mathrm{Li} \text { metal }}$ in diamond-structure $(F d \overline{3} m)$ silicon that initially contains no vacancies, however, $\{4 \mathrm{Li}, V\}$ is energetically favorable in the $P 6_{1} 22$ and $I a \overline{3}$ lower-symmetry tetrahedrally bonded silicon phases. Hence at room temperature it is very likely that $\{4 \mathrm{Li}, V\}$ will form in a range of silicon environments, breaking silicon-silicon bonds and aiding the integration of lithium into the silicon lattice.

We did not find any $\mathrm{Li} / \mathrm{H} / \mathrm{V}$ defects that are more stable than single-impurity-vacancy defects, implying that lithium does not bind to hydrogen when vacancies are present.

A full study with disordered silicon is beyond the scope of this article but we have studied the $\{4 \mathrm{Li}, V\}$ complex in two other tetrahedrally bonded phases of silicon to assess its stability in other silicon environments like those found after the first charge-discharge cycle of a LIB silicon anode. The BC8 phase $^{31}$ (with $I a \overline{3}$ symmetry) has a density of $2.52 \mathrm{~g} \mathrm{~cm}^{-3}$, which is higher than that of diamond-structure silicon $\left(2.3 .2 \mathrm{~g} \mathrm{~cm}^{-3}\right)$, and we find that the formation energy in the type (i) scenario of the $\{4 \mathrm{Li}, V\}$ defect is $-0.02 \mathrm{eV}$ per $\mathrm{Li}$ atom, showing that lithium will "dig-out" silicon vacancies in BC8 silicon. The hypothetical low-energy $P 6_{1} 22$ framework structure $^{32}$ has a lower density of $2.10 \mathrm{~g} \mathrm{~cm}^{-3}$, and the $\{4 \mathrm{Li}, V\}$ defect has a formation energy $-0.05 \mathrm{eV}$ per $\mathrm{Li}$ atom in the type 
(i) scenario. Hence $\{4 \mathrm{Li}, V\}$ is stable over a range of silicon environments with a range of silicon densities and is therefore likely to be stable in disordered silicon phases.

Our calculations show that the $\{4 \mathrm{Li}, V\}$ defect is also stable in diamond-structure $(F d \overline{3} m)$ germanium, with a formation energy of $-0.2 \mathrm{eV}$ per $\mathrm{Li}$ atom, however, due to the smaller lattice constant of diamond, the defect has a much larger formation energy of $\sim 4 \mathrm{eV}$ per $\mathrm{Li}$ atom, and it causes considerable stress within the diamond lattice.

We have presented the structure and electronic properties of the $\{4 \mathrm{Li}, V\}$ complex. The complex is stable once it has formed due to its very low formation energy, and some experimental evidence exists for such a defect. ${ }^{22}$ We attribute the stability of $\{4 \mathrm{Li}, V\}$ to it satisfying the Zintl rules for intermetallic chemical bonding, and other defects such as $\{\mathrm{Li}, V\},\{2 \mathrm{Li}, V\}$, and $\{3 \mathrm{Li}, V\}$ are substantially higher in energy. In scenarios (i) and (ii), the vacancy is formed alongside the inclusion of lithium. This is unlikely to be relevant to the electrochemical lithiation of crystalline silicon. Scenario (iii) is more physically pertinent since monovacancies already exist in silicon and $\{4 \mathrm{Li}, V\}$ can form during lithiation.

The $\{4 \mathrm{Li}, V\}$ defect is stable in a range of tetrahedrally bonded silicon environments, which suggests that it may also be stable in disordered phases of silicon. Scenarios (i) and (ii) show that $\{4 \mathrm{Li}, V\}$ will form on delithiation of $a-\mathrm{Li}_{y} \mathrm{Si}$, where amorphous silicon is formed from high lithium-content lithium silicides which will have a chemical potential similar to bulk lithium. In this case as $a-\mathrm{Li}_{y} \mathrm{Si}$ is delithiated, the silicon recombines to form $a-\mathrm{Si}$, and due to its high stability the $\{4 \mathrm{Li}, V\}$ defect is able to form, trapping both lithium and silicon vacancies in the amorphous silicon. The formation of very stable lithium defects may contribute to incomplete delithiation and a subsequent loss of capacity of a silicon anode. Of course, many other lithium-silicon structures will form during the lithiation of a silicon anode in an LIB, but the $\{4 \mathrm{Li}, V\}$ defect in silicon is an example of a very stable lithium-silicon structure.

This work was supported by the Engineering and Physical Sciences Research Council (EPSRC) of the United Kingdom. E.S. acknowledges support from a Marie Curie Intra-European Fellowship within the 7th European Community Framework Program and thanks Churchill College (Cambridge, UK) for a Sackler Research Fellowship. Computational resources were provided by the University College London Research Computing service.
*Present address: Cambridge University Nanoscience Centre, 11 J. J. Thomson Avenue, Cambridge CB3 OFF, United Kingdom; ajm255@cam.ac.uk

${ }^{1}$ S. Dannefaer and T. Bretagnon, J. Appl. Phys. 77, 5584 (1995).

${ }^{2}$ B. Goldstein, Phys. Rev. Lett. 17, 428 (1966).

${ }^{3}$ O. A. Klimkova and O. R. Niyazova, Phys. Stat. Sol. (a) 3, K93 (1970).

${ }^{4}$ W. Wan, Q. Zhang, Y. Cui, and E. Wang, J. Phys.: Condens. Matter 22, 415501 (2010).

${ }^{5}$ S. Lai, J. Electrochem. Soc. 123, 1196 (1976).

${ }^{6}$ C. J. Wen and R. A. Huggins, J. Sol. Stat. Chem. 37, 271 (1981).

${ }^{7}$ W. J. Weydanz, M. Wohlfahrt-Mehrens, and R. A. Huggins, J. Power Sources 81-82, 237 (1999).

${ }^{8}$ B. Peng, F. Cheng, Z. Tao, and J. Chen, J. Chem. Phys. 133, 034701 (2010).

${ }^{9}$ H. Kim, K. E. Kweon, C.-Y. Chou, J. G. Ekerdt, and G. S. Hwang, J. Phys. Chem. C 114, 17942 (2010).

${ }^{10}$ G. D. Watkins and F. S. Ham, Phys. Rev. B 1, 4071 (1970).

${ }^{11}$ B. Key, R. Bhattacharyya, M. Morcrette, V. Seznéc, J.-M. Tarascon, and C. P. Grey, J. Am. Chem. Soc. 131, 9239 (2009).

${ }^{12}$ J. Li and J. R. Dahn, J. Electrochem. Soc. 154, A156 (2007).

${ }^{13}$ M. K. Y. Chan, C. Wolverton, and J. P. Greeley, J. Am. Chem. Soc. 134, 14362 (2012).

${ }^{14}$ J. L. Goldman, B. R. Long, A. A. Gerwith, and R. G. Nuzzo, Adv. Funct. Mater. 21, 2412 (2011).

${ }^{15}$ A. J. Morris, C. P. Grey, R. J. Needs, and C. J. Pickard, Phys. Rev. B 84, 224106 (2011).

${ }^{16}$ C. J. Pickard and R. J. Needs, Phys. Rev. Lett. 97, 045504 (2006); J. Phys.: Condens. Matter 23, 053201 (2011).
${ }^{17}$ A. J. Morris, C. J. Pickard, and R. J. Needs, Phys. Rev. B 78, 184102 (2008).

${ }^{18}$ A. J. Morris, C. J. Pickard, and R. J. Needs, Phys. Rev. B 80, 144112 (2009).

${ }^{19}$ J. Mulroue, A. J. Morris, and D. M. Duffy, Phys. Rev. B 84, 094118 (2011).

${ }^{20}$ J. Mulroue, M. Watkins, A. J. Morris, and D. Duffy, J. Nucl. Mat. 437, 261 (2012).

${ }^{21}$ S. J. Clark et al., Z. Kristallogr. 220, 567 (2005).

${ }^{22}$ L. Canham, G. Davies, E. C. Lightowlers, and G. W. Blackmore, Physica B 117, 119 (1983).

${ }^{23}$ C. J. Pickard and F. Mauri, Phys. Rev. B 63, 245101 (2001).

${ }^{24}$ J. R. Yates, C. J. Pickard, and F. Mauri, Phys. Rev. B 76, 024401 (2007).

${ }^{25}$ A. S. Ivanov, A. J. Morris, K. V. Bozhenko, C. J. Pickard, and A. I. Boldyrev, Angew. Chem. Int. Ed. 51, 8330 (2012).

${ }^{26}$ A. J. Morris, R. J. Nicholls, C. J. Pickard, and J. R. Yates, OPTADOS User Guide, Version 1.0.0 (University of Cambridge, Cambridge, 2013); R. J. Nicholls, A. J. Morris, C. J. Pickard, and J. R. Yates, J. Phys.: Conf. Ser. 371, 012062 (2012).

${ }^{27}$ M. J. Puska, S. Pöykkö, M. Pesola, and R. M. Nieminen, Phys. Rev. B 58, 1318 (1998).

${ }^{28}$ J. Lento and R. M. Nieminen, J. Phys.: Condens. Matter 15, 4387 (2003).

${ }^{29}$ M. I. J. Probert and M. C. Payne, Phys. Rev. B 67, 075204 (2003).

${ }^{30}$ F. Corsetti and A. A. Mostofi, Phys. Rev. B 84, 035209 (2011).

${ }^{31}$ R. H. Wentorf and J. S. Kasper, Science 139, 338 (1963).

${ }^{32}$ C. J. Pickard and R. J. Needs, Phys. Rev. B 81, 014106 (2010). 